\title{
Intact Dirac Cones at Broken Sublattice Symmetry: Photoemission Study of Graphene on Ni and Co
}

\author{
A. Varykhalov, ${ }^{1}$ D. Marchenko, ${ }^{1}$ J. Sánchez-Barriga, ${ }^{1}$ M. R. Scholz,${ }^{1}$ B. Verberck, ${ }^{2}$ \\ B. Trauzettel, ${ }^{3}$ T. O. Wehling, ${ }^{4,5}$ C. Carbone, ${ }^{6}$ and O. Rader ${ }^{1}$ \\ ${ }^{1}$ Helmholtz-Zentrum Berlin für Materialien und Energie, Elektronenspeicherring BESSY II, \\ Albert-Einstein-Strasse 15, D-12489 Berlin, Germany \\ ${ }^{2}$ Departement Fysica, Universiteit Antwerpen, Groenenborgerlaan 171, B-2020 Antwerpen, Belgium \\ ${ }^{3}$ Institut für Theoretische Physik und Astrophysik, Universität Würzburg, Am Hubland, D-97074 Würzburg, Germany \\ ${ }^{4}$ Institut für Theoretische Physik, Universität Bremen, Otto-Hahn-Allee 1, D-28359 Bremen, Germany \\ ${ }^{5}$ Bremen Center for Computational Materials Science, Am Fallturm 1a, D-28359 Bremen, Germany \\ ${ }^{6}$ Istituto di Struttura della Materia-Consiglio Nazionale delle Ricerche, Basovizza, I-34149 Trieste, Italy
}

(Received 3 February 2012; published 20 December 2012)

\begin{abstract}
The appearance of massless Dirac fermions in graphene requires two equivalent carbon sublattices of trigonal shape. While the generation of an effective mass and a band gap at the Dirac point remains an unresolved problem for freestanding extended graphene, it is well established by breaking translational symmetry by confinement and by breaking sublattice symmetry by interaction with a substrate. One of the strongest sublattice-symmetry-breaking interactions with predicted and measured band gaps ranging from $400 \mathrm{meV}$ to more than $3 \mathrm{eV}$ has been attributed to the interfaces of graphene with $\mathrm{Ni}$ and $\mathrm{Co}$, which are also promising spin-filter interfaces. Here, we apply angle-resolved photoemission to epitaxial graphene on $\mathrm{Ni}$ (111) and $\mathrm{Co}(0001)$ to show the presence of intact Dirac cones $2.8 \mathrm{eV}$ below the Fermi level. Our results challenge the common belief that the breaking of sublattice symmetry by a substrate and the opening of the band gap at the Dirac energy are in a straightforward relation. A simple effective model of a biased bilayer structure composed of graphene and a sublattice-symmetry-broken layer, corroborated by densityfunctional-theory calculations, demonstrates the general validity of our conclusions.
\end{abstract}

DOI: 10.1103/PhysRevX.2.041017

\section{INTRODUCTION}

The charge carriers in graphene exhibit linear quasirelativistic dispersion and form two conical bands $\pi$ and $\pi^{*}$ that touch each other at the corners of the surface Brillouin zone and at one particular energy, referred to as the Dirac energy $\left(E_{\mathrm{D}}\right)[1-4]$. In freestanding graphene, charge neutrality aligns Dirac and Fermi energies, and a Fermi surface emerges, composed of only six points $\left(3 \times \bar{K}\right.$ and $\left.3 \times \bar{K}^{\prime}\right)$ in the two-dimensional Brillouin zone. Because of this compact Fermi surface and a strong interplay between structural and electronic properties, graphene is extraordinarily promising for applications in electronic devices: Quantum mechanics dictates that the linear bands in the vicinity of the Dirac energy are very sensitive to external perturbations of the graphene lattice. For freestanding extended graphene, the generation of effective mass and band gap at $E_{\mathrm{D}}$ is still unresolved [4,5] but it is well established by the breaking of either translational symmetry by confinement [6-8] or of sublattice symmetry by interaction with a substrate [9-16]. This dependence is strong and can be employed for steering the band gap and, in turn, for

Published by the American Physical Society under the terms of the Creative Commons Attribution 3.0 License. Further distribution of this work must maintain attribution to the author(s) and the published article's title, journal citation, and DOI.
Subject Areas: Condensed Matter Physics, Graphene

controlling electric transport. This idea, flavored by an exceptionally high mobility of the quasirelativistic charge carriers, has already led to the creation of ultrafast carbonbased transistors [17,18]. In the present work, we question the widely acknowledged strength of the interrelation between band gap and symmetry breaking in epitaxial graphene and present two examples where such interrelation is missing.

Because of the honeycomblike atomic structure consisting of two equivalent sublattices $A$ and $B$, the Hamiltonian for graphene acts on a two-component spinor $\Psi=\left(\Psi_{A}, \Psi_{B}\right)$ :

$$
H=\left(\begin{array}{cc}
\frac{\Delta}{2} & \nu\left(k_{x}-i k_{y}\right) \\
\nu\left(k_{x}+i k_{y}\right) & -\frac{\Delta}{2}
\end{array}\right) .
$$

The parameter $\Delta$ stands here for the asymmetry of the electric potential on sites at carbon atoms of sublattices $A$ and $B[1,4,10,12]$.

Should there be no asymmetry $(\Delta=0)$, the solution of the Schrödinger equation implies a Dirac equation with linear conical dispersion $\mathrm{E}(\mathbf{k})=\nu k$. In the opposite case, when $A$ and $B$ sites are inequivalent and $\Delta \neq 0$, the physical picture of massless relativistic quasiparticles is expected to break down and a band gap of size $\Delta$ should open exactly at the Dirac energy. The breaking of $A-B$ symmetry as the origin of the band gap in graphene is widely acknowledged [9-16]. 
The Dirac bands react very sensitively to a reduction of $A-B$ symmetry. An example is the band structure of graphene placed on hexagonal boron nitride. Density functional theory (DFT) shows that, in the case of physisorption with a very large ( $>3 \AA$ ) interlayer distance, the weak sublattice asymmetry induces a substantial band gap in graphene at $E_{\mathrm{D}}$ of $>50 \mathrm{meV}$ [11].

There are systems in which the deterioration of the $A-B$ symmetry is by far stronger than in the example given above. These are graphene on transition-metal substrates $\mathrm{Ni}(111)$ and $\mathrm{Co}(0001)$ [14,15,19-23]. Surface lattice constants of graphene and fcc $\mathrm{Ni}$ and hcp Co are almost equal (mismatches of $1.2 \%$ and $1.9 \%$, respectively). Such minor mismatches render in situ grown graphene in registry with these substrates. It has been noticed for $\mathrm{Ni}(111)$ that the graphene growth from the gas phase is a self-limiting process that terminates after a single graphene layer because of vanishing reactivity [24]. The crystal structures of both systems are well established by quantitative $(I-V)$ low-energy electron diffraction [20], scanning tunneling microscopy (STM) [25], and DFT [14,25-27] and are nearly equivalent: Carbon atoms from sublattice $A$ are placed on top of $\mathrm{Ni}(\mathrm{Co})$ atoms belonging to the topmost atomic layer of the substrate, while atoms from sublattice $B$ are located between them above the substrate hollow sites [Figs. 1(b) and 1(d)]. In terms of the $\mathcal{A} \mathcal{B C}$ notation for stacking [25], such placement is referred to as the $\mathcal{A C}$ configuration. In view of the strong chemical interaction between graphene and $\mathrm{Ni}(\mathrm{Co})$ and the small (2.11 \pm $0.07 \AA$ [20]) separation between them, the sublattice asymmetry is enormous. The nonequivalence of $A$ and $B$ sites is seen clearly in our STM images of graphene on $\mathrm{Ni}$ and Co in Figs. 1(a) and 1(c). In fact, only one graphene sublattice is seen, resulting in a threefold instead of a sixfold symmetry. The reason for this effect is a strong variation of the local density of states between the sublattices due to the substrate. The fact that we observe threefold symmetric STM images at very low bias voltages $\left(V_{t}<+20 \mathrm{mV}\right)$ is remarkable and supports the $\mathcal{A C}$ configuration of stacking. Such proof has, e.g., succeeded with graphene on $\mathrm{SiC}$ [28]. The more symmetric $\mathcal{B C}$ configuration (where substrate atoms from the topmost atomic layer are located under the centers of graphene hexagons [29]) can be ruled out, since, for such a structure, low-bias STM images show both graphene sublattices as equally intense [30].

The $\mathcal{A C}$ configuration of stacking in combination with a strong interfacial chemical interaction plausibly suggests that graphene on fcc Ni and hcp Co must have a large band gap at the Dirac energy twice the size of the modulation potential. Indeed, the Dirac point has been reported to be destroyed [19], and large band gaps of 0.4 to $0.8 \mathrm{eV}$ for graphene on $\mathrm{Ni}(111)$ [14] and $\gg 1 \mathrm{eV}$ for graphene on Co(0001) [19] have been predicted by DFT. With van der Waals corrections, the optimum interlayer distance between graphene and $\mathrm{Ni}(111)$ of $2.1 \AA$ is confirmed and a
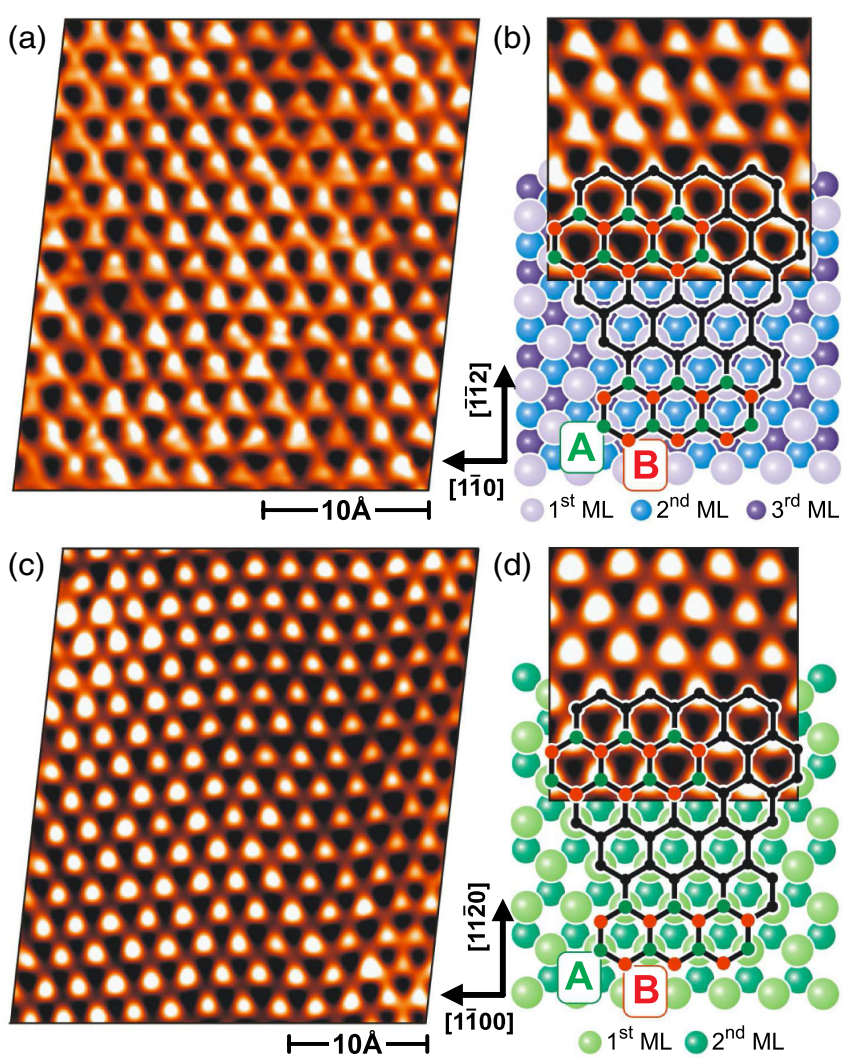

FIG. 1. STM evidence for the sublattice symmetry breaking of graphene (a),(b) on $\mathrm{Ni}(111)$ and (c),(d) on $\mathrm{Co}(0001)$. STM images in (a) and (c) show pronounced threefold symmetry. (b),(d) display structural models of graphene on $\mathrm{Ni}$ and $\mathrm{Co}$, respectively, superimposed on the STM data. $A-B$ sublattice symmetry breaks due to $\mathcal{A C}$ stacking, causing different environments for $A$ and $B$ sublattices of graphene (green and red nodes, respectively). Tunneling parameters: $V_{t}=+2 \mathrm{mV}, I_{t}=25 \mathrm{nA}$.

band gap $>3 \mathrm{eV}$ is obtained [31]. While early angle-resolved photoemission (ARPES) experiments of graphene on $\mathrm{Ni}(111)$ did not report a band gap between $\pi$ and $\pi^{*}$ states at $\bar{K}[24,32]$, recent experiments give different results. The interaction between graphene and $\mathrm{Ni}(111)$ can be strongly reduced by potassium intercalation [15]. For graphene on $\mathrm{Ni}(111)$ without the potassium, a tight-binding model has been fitted to the experimental data and shows a gap of $0.9 \mathrm{eV}$ due to sublattice symmetry breaking [15]. A similar decoupling is achieved by intercalation of $\mathrm{Al}$ [33], but, for graphene on $\mathrm{Ni}(111)$ without the Al layer, the Dirac cone has been reported not to be preserved but destroyed [33,34].

\section{EXPERIMENTAL RESULTS}

In this paper, we accurately examine the electronic structure of graphene on $\mathrm{Ni}(111)$ and $\mathrm{Co}(0001)$ by means of ARPES and demonstrate that, despite a strong chemical interaction with the substrates (resulting in strong $n$ doping and hybridization) and strong $A-B$ symmetry breaking, the linear bands of Dirac fermions remain intact and no gap opens at the Dirac energy. Our observations strongly 

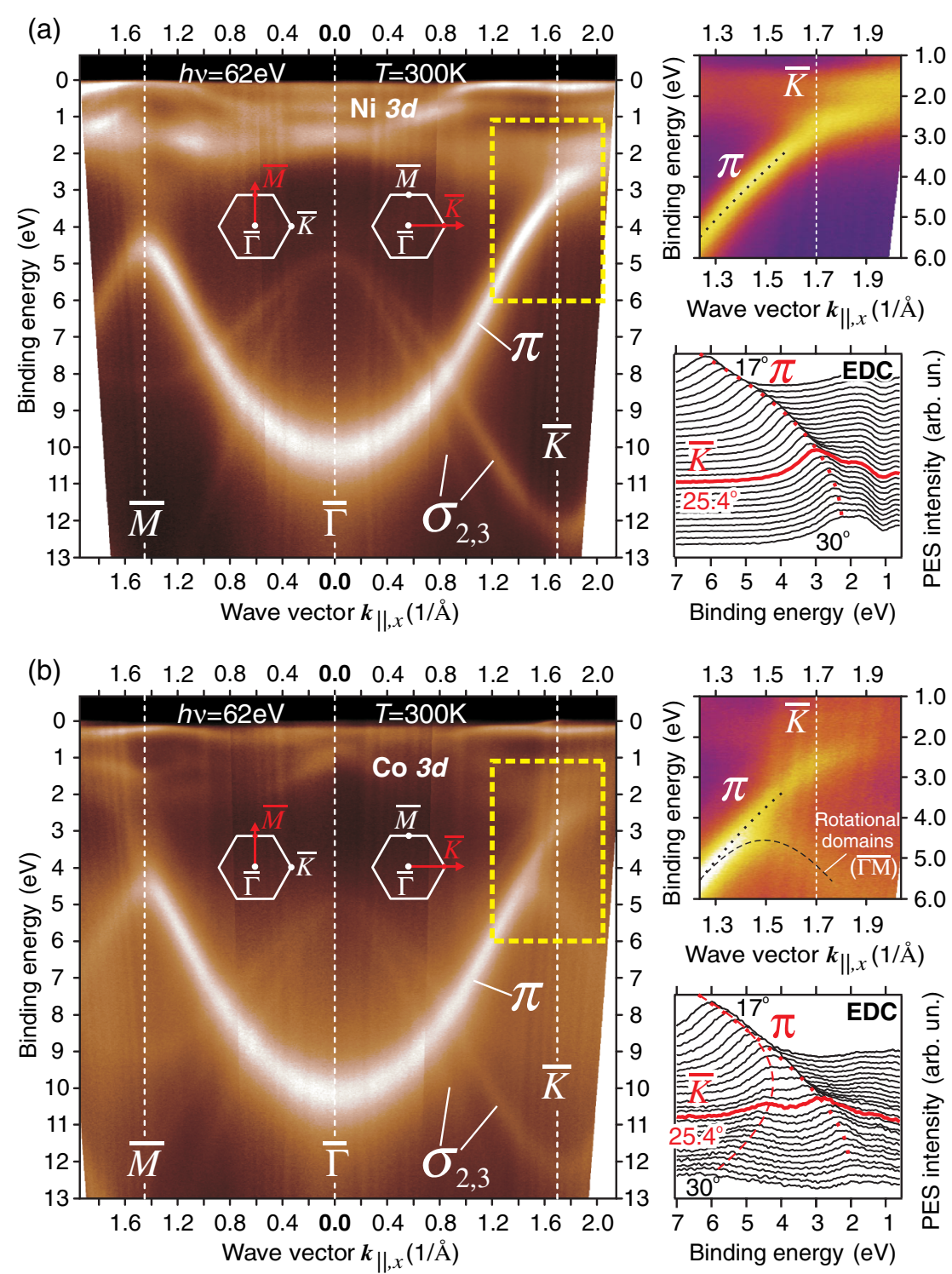

FIG. 2. ARPES characterization of the gapless dispersion through the $\bar{K}$ point from $\bar{\Gamma}$. Overall band structures of graphene on (a) $\mathrm{Ni}(111)$ and on (b) $\mathrm{Co}(0001)$ measured along the $\overline{\Gamma M}$ and $\overline{\Gamma K}$ directions of the surface Brillouin zone. The insets on the right-hand side of (a) and (b) emphasize the gapless dispersion of the $\pi$ band at the $\bar{K}$ point.

contradict the structural models established for graphene on fcc $\mathrm{Ni}(111)$ and hcp $\mathrm{Co}(0001)$ by theoretical and experimental studies in recent decades and prompt a fundamental question: Is sublattice symmetry breaking a sufficient condition for the band gap in substrate-supported graphene?

Figure 2 shows the overall band structures of graphene grown on Ni(111) [Fig. 2(a)] and Co(0001) [Fig. 2(b)] sampled along the $\overline{\Gamma M}$ and $\overline{\Gamma K}$ lines of the surface Brillouin zone of graphene. In both cases, the interaction with the substrate shifts the $\pi$ band to higher binding energies: Its bottom at the $\bar{\Gamma}$ point is located at a much higher binding energy (approximately $10.1 \mathrm{eV}$ ) as compared to freestanding graphene (approximately $8.4 \mathrm{eV}$ ). The intriguing behavior of the $\pi$ band is observed close to the $\bar{K}$ point, the exact location of which is determined from the backfolding of the $\sigma_{2,3}$ band at the zone boundary. One clearly sees that the $\pi$ band disperses upward into the second Brillouin zone and that no band gap is visible at the $\bar{K}$ point. Bands of graphene on $\mathrm{Co}(0001)$ [Fig. 2(b)] exhibit similar behavior but with additional contributions from non- $\mathrm{R} 0^{\circ}$ rotational variants. The regions of interest (dashed yellow area) are additionally magnified in the insets and emphasized with a different color scale as well as by the stacking of spectra [energy distribution curves (EDCs)]. The full scenario of the $\pi$-band dispersion cannot be seen from these data because the opposite side of the $\pi$ band is suppressed by the destructive interference of photoelectrons along $\overline{\Gamma K}$ [35].

This effect is visible in a full photoemission mapping of the $\pi$ band. Results for graphene on $\mathrm{Ni}(111)$ are presented 


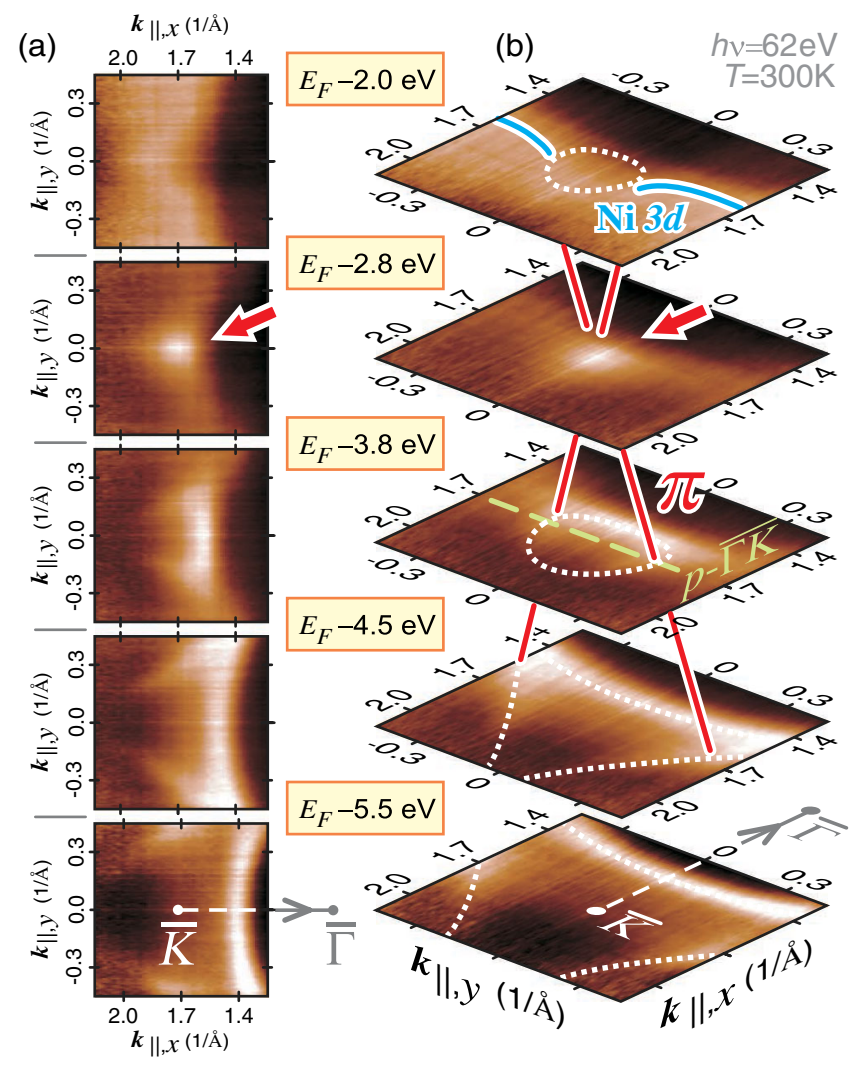

FIG. 3. Full ARPES mapping of the $\pi$ band in graphene on $\mathrm{Ni}(111)$ around $\bar{K}$. (a) Constant energy surfaces reveal characteristic triangular contours of the $\pi$ band. The Dirac point (red arrow) is clearly observable at $2.8 \mathrm{eV}$ binding energy. (b) Same as (a) but in isometric representation and with a threedimensional sketch of the band structure.

in Fig. 3. Figure 3(a) shows several constant energy surfaces sliced at various binding energies. At high binding energies, one notes characteristic triangular contours of the $\pi$ band that fit well with the tight-binding band structure of graphene [1]. At $2.8 \mathrm{eV}$, the conical band converges remarkably in one single spot (red arrow in Fig. 3) that corresponds to the Dirac point and indicates the presence of an intact Dirac cone. For lower binding energies $<2 \mathrm{eV}$, no contours of the graphene band are seen, except for the $3 d$ bands of Ni. Figure 3(b) displays an isometric representation of constant energy surfaces with dispersions of the $\pi$ band and Ni $3 d$ states, which are highlighted with lines. Full mapping for graphene on $\operatorname{Co}(0001)$ (not shown here) also reveals an intact Dirac point.

When the dispersion is sampled along the direction perpendicular to $\overline{\Gamma K}[p-\overline{\Gamma K}$ : long-dashed green line in the middle layer of Fig. 3(b)], the interference effects in the photoemission final state are equal and both sides of the Dirac cone appear. This direction is presented in Figs. 4(a)-4(d) for graphene on $\mathrm{Ni}(111)$ and in Figs. 4(e) and 4(f) for graphene on $\mathrm{Co}(0001)$. In order to emphasize the dispersion, we show color plots of the second derivative $\partial^{2} I\left(E, k_{\|}\right) / \partial E^{2}$ of the photoemission signal. Stacked spectra in Fig. 4 represent
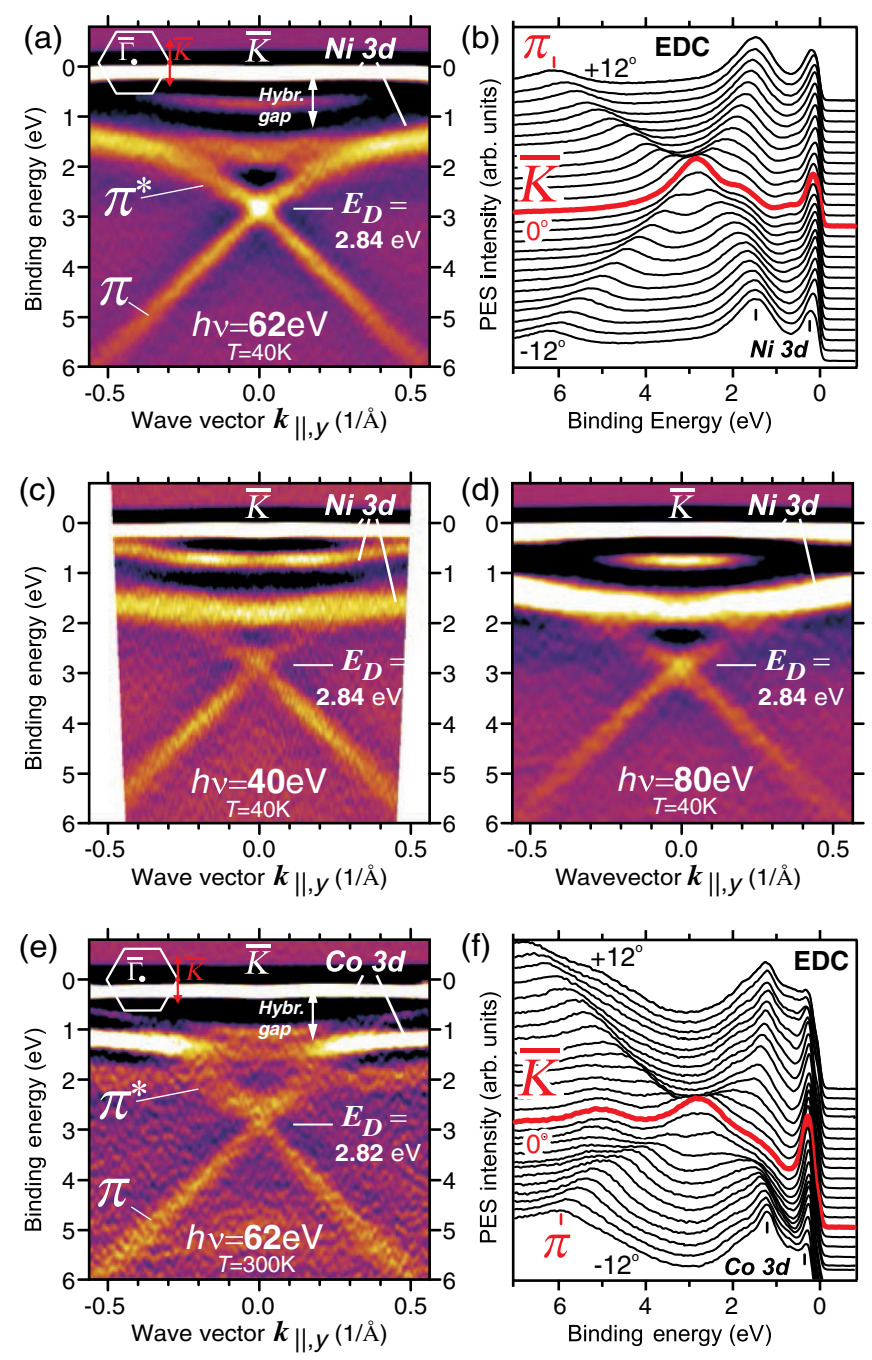

FIG. 4. Direct observation of intact Dirac cones measured perpendicular to the $\overline{\Gamma K}$ direction. (a),(b) Dirac cone in graphene on $\mathrm{Ni}(111)$ measured at $h \nu=62 \mathrm{eV}$ (color plot and EDCs). (c), (d) Dirac cone in graphene on $\mathrm{Ni}(111)$ sampled at $h \nu=40$ and $80 \mathrm{eV}$. (e),(f) Intact Dirac cone measured in graphene on $\mathrm{Co}(0001)$ at $h \nu=62 \mathrm{eV}$ (color plot and EDCs).

raw data. Figures 4(a) and 4(b) report photoemission from graphene on $\mathrm{Ni}(111)$ acquired at $h \nu=62 \mathrm{eV}$. The intact Dirac cone is evident: Dispersion of the $\pi$ band is linear for binding energies higher than $E_{\mathrm{D}}$, and no band gap is seen at the Dirac crossing point, despite the broken sublattice symmetry of graphene. The Dirac energy is found at $2.84 \mathrm{eV}$, consistent with strong $n$ doping. For binding energies lower than $E_{\mathrm{D}}$, the dispersion of the $\pi$ band is not linear anymore. Apparently, it becomes renormalized due to hybridization with $3 d$ bands of Ni. This hybridization also leads to the formation of a hybridization band gap in the antibonding band $\pi^{*}$ for binding energies less than $1.4 \mathrm{eV}$. This gap, which has been noticed in all previous studies $[15,24,32,34]$, is, however, of trivial nature: It does not emerge at $E_{\mathrm{D}}$ and is not related to the broken symmetry of graphene. 
In order to exclude a possibly "hidden" band gap at $E_{\mathrm{D}}$ by a superimposed photoemission signal from $\mathrm{Ni} 3 d$ states, we have sampled the Dirac cone at various photon energies. Results for $h \nu=40 \mathrm{eV}$ and $h \nu=80 \mathrm{eV}$ are shown in Figs. 4(c) and 4(d), respectively. While the bulk Ni bands indeed disperse with $\mathbf{k}_{\perp}$, the Dirac cone does not change, which means that the Dirac cone cannot be a Ni bulk feature.

High-resolution ARPES data for graphene on $\mathrm{Co}(0001)$ are shown in Figs. 4(e) and 4(f). Here, we also observe an intact Dirac cone, in spite of the broken $A-B$ symmetry of graphene. The Dirac-point binding energy of graphene on the Co surface is almost equal to that from Ni: $E_{\mathrm{D}}=2.82 \mathrm{eV}$. Moreover, at $h \nu=62 \mathrm{eV}$, the Co $3 d$ bands emerge at lower binding energies $(1.2 \mathrm{eV})$, as compared to $\mathrm{Ni}(111)$. We can see that the $\pi^{*}$ band is also linear, with a group velocity equal to the one of the $\pi$ band. Graphene on $\mathrm{Ni}$ and Co leads to perfect spin filters [14]. Indeed, Co has a large magnetic exchange splitting of $1.4 \mathrm{eV}$, on average, and the peak at $1.2 \mathrm{eV}$ is assigned to minority spin since the majority-spin peaks are strongly broadened and usually difficult to observe. Such difference between minority and majority states means that, for higher binding energies than the hybridization point of $1.2 \mathrm{eV}$, the $\pi$ states of graphene are expected to become strongly spin polarized. One candidate for the corresponding Co majority-spin band is the weak band appearing around $2.0 \mathrm{eV}$, which hybridizes with the $\pi^{*}$ band as well. This assignment is, however, not definite since the separation is much less than $1.4 \mathrm{eV}$.

Our main message is that, with the exception of the emergence of this hybridization gap in the $\pi^{*}$ band that is well separated from $E_{\mathrm{D}}$ and of the strong binding-energy shift, the band structures of graphene on $\mathrm{Ni}(111)$ and $\mathrm{Co}(0001)$ can be similar to those of freestanding graphene. The simplest explanation for the existence of intact Dirac cones is that in reality the sublattice symmetry in graphene on $\mathrm{Ni}(111)$ and $\mathrm{Co}(0001)$ is not broken and the established $\mathcal{A C}$ configuration of stacking [Figs. 1(b) and 1(d)] is not realized. The only possibility for a much weaker distortion
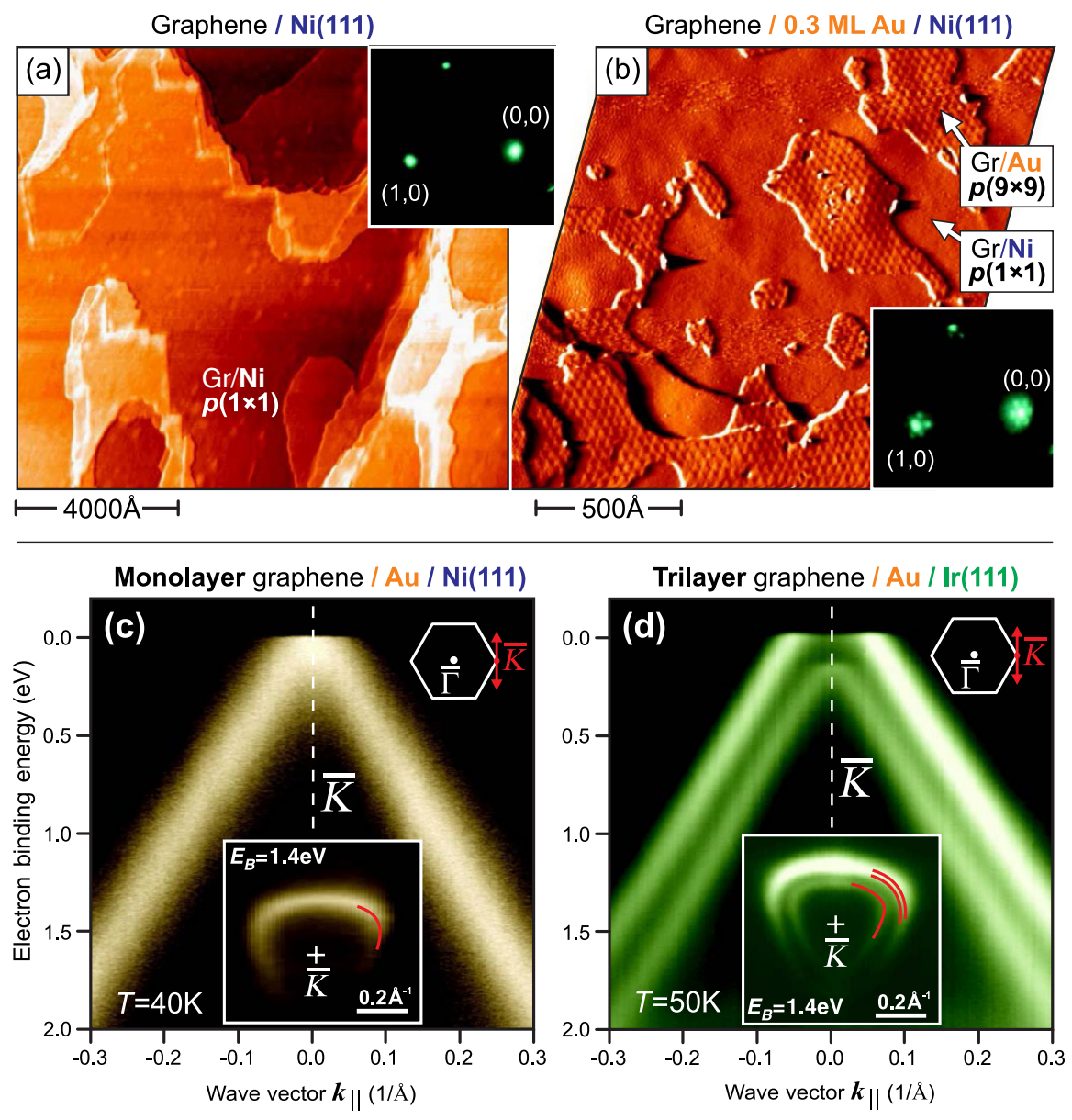

FIG. 5. Absence of graphene superstructure and multilayer formation. (a) Large-scale STM characterization of graphene on Ni(111) showing no moiré superstructure due to lattice mismatch. (b) The intermediate-scale STM image of graphene on Ni(111) partially intercalated with $\mathrm{Au}$ (image contrast is enhanced) reveals a $p(9 \times 9)$ moiré above Au islands but no moiré pattern in the graphene on $\mathrm{Ni}$ areas. The insets represent LEED images $(E=40 \mathrm{eV})$. (c) The electronic structure of graphene decoupled from Ni(111) by intercalation of Au reveals only one Dirac cone, which means that graphene is a monolayer. (d) Control sample [graphene trilayer on $\operatorname{Ir}(111)]$ shows characteristic trilayerlike bands after intercalation of $\mathrm{Au}$. 
of the sublattice symmetry is the $\mathcal{B C}$ configuration of stacking, which was already proposed by Rosei et al. in 1983 [29]. The model by Rosei et al. suggests, however, that graphene on $\mathrm{Ni}(111)$ is just physisorbed, with large interfacial separation (approximately $2.8 \AA$ ), and has no chemical interaction with the substrate. Both are in obvious contradiction to our STM data and to the observation of hybridization between the $\pi^{*}$ band and the $d$ bands of Ni. The $\mathcal{B C}$ model by Rosei et al. has indeed been disproved by many experimental and theoretical studies $[14,20,26,27]$ in favor of $\mathcal{A C}$ stacking.

We took particular care to test how well graphene is in registry to $\mathrm{Ni}(111)$ in our samples. One might speculate that the small lattice mismatch of $1.2 \%$ between graphene and Ni may cause a long-range [order of $p(100 \times 100)]$ moiré pattern, resulting in an effective cancellation of the sublattice-symmetry-breaking effect [36,37]. Our samples have been extensively characterized by STM at an enlarged scale. One such measurement is shown in Fig. 5(a) and reveals no moiré pattern corresponding approximately to a $p(100 \times 100)$ superstructure. To exclude the possibility that such a moiré structure is not resolved because of poor lateral resolution of the STM tip, we have measured graphene on $\mathrm{Ni}(111)$ partially intercalated with Au [Fig. 5(b)]. One can see that the sharp $p(9 \times 9)$ moiré pattern of graphene on $\mathrm{Au} / \mathrm{Ni}(111)$ [21] is clearly resolved in graphene above $\mathrm{Au}$ while nonintercalated areas between $\mathrm{Au}$ islands remain structureless. Zooming the STM to atomic resolution over an arbitrary site of the nonintercalated area delivers an image with threefold symmetry like that in Fig. 1(a), while a moiré superstructure would cause the periodic appearance of sixfold symmetry. Also, LEED patterns of graphene on $\mathrm{Ni}(111)$ do not indicate the presence of any moiré superstructure (down to $15 \mathrm{eV}$ ). We are able to conclude that our graphene samples are perfectly in registry with $\mathrm{Ni}(111)$. We have also verified that this structural coherence does not change with the temperature as no change in the Dirac point occurs by continuous cooling (down to $20 \mathrm{~K}$ ) and warming up (to $400 \mathrm{~K}$ ) of the samples.
We have also verified that our $\mathrm{Ni}(\mathrm{Co})$ samples bear only one graphene layer on top. The self-limitation of the chemical-vapor deposition (CVD) in ultrahigh vacuum by growth of a single layer of graphene is well established $[24,38]$. One might speculate that a bilayer could emerge due to segregation of carbon from the substrate. In this case, the intact Dirac cones seen in ARPES would originate from the topmost layer, while the band structure of the underlying graphene layer can be destroyed by interaction with Ni (Co). There are two arguments against such a scenario. The first argument is that the strong $n$ doping of the Dirac cone and its hybridization with Ni $d$ states mean that the graphene layer with the Dirac cone has a direct interface to the Ni substrate. The second argument comes from an intercalation experiment comparing ARPES data from our graphene-on-Ni(111) samples to those from a control sample that carries few-layer graphene. As control sample, we have chosen $\operatorname{Ir}(111)$, on which a graphene trilayer has been fabricated by segregation of carbon [39]. The graphene overlayers of both samples have been decoupled from their substrates in an identical manner by intercalation of a Au monolayer [40]. Figures 5(c) and 5(d), respectively, display band structures of graphene-on-Ni and graphene-trilayer-on-Ir samples acquired by ARPES after intercalation of Au. For the control sample, a characteristic trilayer band structure is seen, while for the graphene on $\mathrm{Au} / \mathrm{Ni}$ sample, only one Dirac cone is observed, which proves that graphene initially synthesized on $\mathrm{Ni}(111)$ is a monolayer.

\section{THEORETICAL RESULTS}

The coexistence of $A-B$ symmetry breaking and intact Dirac cones can be analyzed by means of a simple and intuitive model valid in the vicinity of $\bar{K}$. By representing the top layer of the $\mathrm{Ni}(\mathrm{Co})$ substrate by a boron-nitridelike monolayer with boron sites occupied by $\mathrm{Ni}(\mathrm{Co})$ atoms and nitrogen sites by vacancies (V) [Fig. 6(a)], the simplest extension of the $2 \times 2$ Hamiltonian (1) is the following $4 \times 4$ Hamiltonian:

$$
H=\left(\begin{array}{cc}
E_{\mathrm{C}} & \nu_{\mathrm{C}}\left(k_{x}+i k_{y}\right) \\
\nu_{\mathrm{C}}\left(k_{x}-i k_{y}\right) & E_{\mathrm{C}} \\
t^{\prime} & 0 \\
0 & 0
\end{array}\right.
$$

$$
\left.\begin{array}{cc}
t^{\prime} & 0 \\
0 & 0 \\
E_{s}-e W & \nu_{s}\left(k_{x}+i k_{y}\right) \\
\nu_{s}\left(k_{x}-i k_{y}\right) & E_{\mathrm{V}}-e W
\end{array}\right),
$$

where $s$ stands for $\mathrm{Ni}(\mathrm{Co})$. The graphene sheet and the top substrate layer (both with in-plane nearest-neighbor distance $a$ ) are in Bernal stacking with respect to each other, corresponding to the $\mathcal{A C}$ configurations observed by STM (Fig. 1). The quantities $\nu_{\mathrm{C}}$ and $\nu_{s}$ are related to the in-plane hopping parameters $t_{\mathrm{C}}$ and $t_{s}$ for the graphene and substrate layers, respectively, via $\nu_{\mathrm{C}}=\frac{3}{2} a t_{\mathrm{C}}$ and $\nu_{s}=\frac{3}{2} a t_{s} \cdot t^{\prime}$ represents hopping between the two layers. $E_{\mathrm{C}}, E_{s}$, and $E_{\mathrm{V}}$ are on-site energies. $e W$ is an energy difference between the layers that, in a different experiment, could be associated with a bias voltage $W$. Depending on the values of the energy parameters, the Hamiltonian (2) leads to various qualitatively different band structures. A general observation is that, for a large energy difference $e W$ (mathematically, $\left.\left|e W+E_{\mathrm{C}}-E_{s}\right| \gg t^{\prime}\right)$, an almost vanishing gap $\Delta E$ [decreasing with $|W|$ as $t^{\prime 2} /\left(e W+E_{\mathrm{C}}-E_{s}\right)^{2}$ ] separating almost linear dispersion branches is obtained, as illustrated in Figs. 6(b) and 6(c) for $W=0$ and $W=-4 \mathrm{~V}$, 

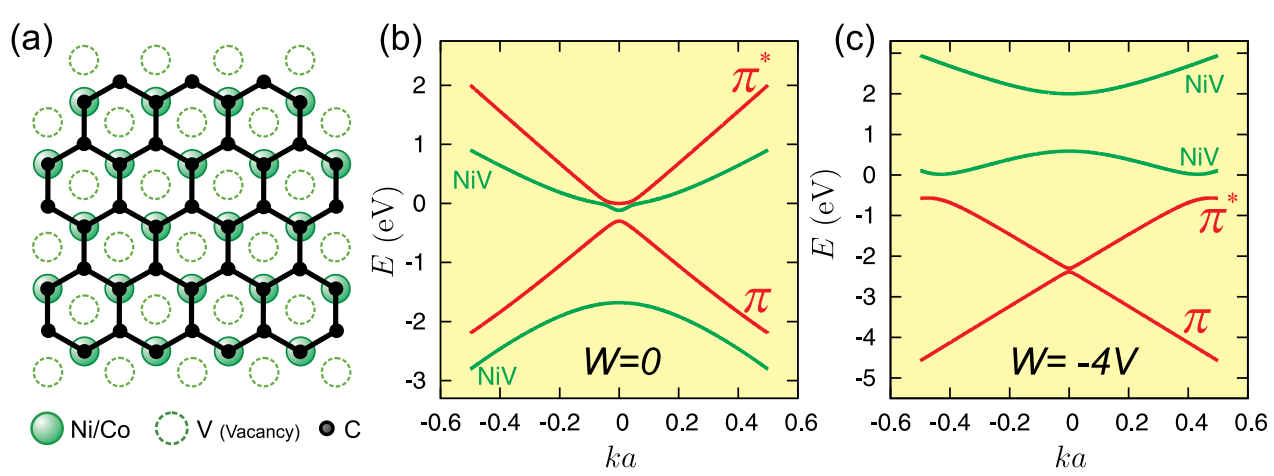

FIG. 6. Diminishing band gap in a simplified model for graphene on $\mathrm{Ni}(111)$ or $\mathrm{Co}(0001)$. The model in geometry (a) corresponds to Eq. (2) and holds near $\bar{K}$. The hopping and on-site energy parameters are assigned typical values: $E_{\mathrm{C}}=-0.3 \mathrm{eV}, E_{s}=-1.5 \mathrm{eV}$, $E_{\mathrm{V}}=0, t_{\mathrm{C}}=3 \mathrm{eV}, t_{s}=2 \mathrm{eV}$, and $t^{\prime}=0.5 \mathrm{eV}$. In (b) and (c), $W$ is set to 0 and $-4 \mathrm{~V}$, respectively, illustrating the emergence of almost linear dispersion in the case of a large energy difference $e W$.

respectively, with typical values for the hopping and onsite energy parameters $E_{\mathrm{C}}, E_{s}, E_{\mathrm{V}}, t_{\mathrm{C}}, t_{s}$, and $t^{\prime}$. For $e W \leq$ $-\left|E_{\mathrm{C}}-E_{s}\right|$, the type of band structure shown in Fig. 6(c) is obtained. The almost-parabolic bands (green curves) are associated with the $\mathrm{NiV}(\mathrm{CoV})$ layer and the almost linear dispersions (red curves) with the graphene layer, reducing to parabolic (massive) and linear (massless) bands, respectively, in the case of the vanishing interlayer coupling $t^{\prime}$. It is seen that a band gap opens at the Dirac point due to the influence of the boron-nitride-like layer that breaks $A-B$ symmetry in the graphene layer. However, the case of the large energy difference $e W$ [Fig. 6(c)] coincides very well with the experimentally observed dispersions (Fig. 4), including the band gaps in the graphene sheet occurring far away from $\bar{K}$. Within our simple model, it follows that a large energy difference $e W$ results in an arbitrarily small band gap and hyperboloidal bands arbitrarily close to Dirac cones in the energy spectrum of graphene on $\mathrm{Ni}(111)$ or $\mathrm{Co}(0001)$ surfaces.

As for physical mechanisms compatible with our simple four-band model, doping of the graphene layer by the metallic $\mathrm{NiV}(\mathrm{CoV})$ layer is a plausible candidate. In this scenario, strong doping corresponds to the large energy difference $e W$ and is responsible for the experimentally observed Dirac conelike feature in the valence band. Another, less trivial, physical explanation for the nearly conserved Dirac cones relies on the hybridization of the $\mathrm{Ni}$ (Co) $3 d$ bands with the C $2 p_{z}$ bands. We have investigated the latter possibility by means of DFT calculations, where we simulate graphene on $\mathrm{Ni}(111)$ by a slab consisting of $15 \mathrm{Ni}$ layers with adsorbed graphene in the $\mathcal{A C}$ stacking configuration using the Vienna $a b$ initio simulation package (VASP) [41,42]. (Further technical details of the DFT calculations have been given elsewhere [43].) The band structure we obtain from our DFT calculations (Fig. 7) is, as such, in agreement with previous results $[26,27,33,43]$.

However, one aspect has been overlooked, so far. There are two Dirac cones near $\bar{K}$ : one above the Fermi level $(E>0)$ and one at $E \approx-3 \mathrm{eV}$. Analyzing the band character of the Dirac feature near $-3 \mathrm{eV}$ in Fig. 7(b), we find spectral weight in sublattice $A$ (carbon atoms with a $\mathrm{Ni}$ atom directly underneath, red lines) predominantly at lower energies than weight from sublattice $B$ [carbon atoms above a hollow site of the $\mathrm{Ni}(111)$ surface, green lines]: The red features are below the green feature at $\bar{K}$. This band structure indicates a certain breaking of the sublattice symmetry. At the Dirac point above the Fermi level, the same holds, but with the opposite sign of the sublattice symmetry breaking: For the Dirac point at $E>0$, the red bands are above the green bands at $\bar{K}$. So, there is sublattice symmetry breaking that must stem from dynamic hybridization effects, as it changes sign with energy. Such dynamic effects can also explain the appearance of two Dirac cones at $\bar{K}$ and trace back to the interaction of $3 d$ states from the topmost $\mathrm{Ni}$ atoms with the graphene layer: For both Dirac cones, our DFT calculations reveal a significant admixture of $3 d$ states from the topmost Ni atoms [Fig. 7(c)]. Well in line with the effective model of Eq. (2), the splitting between the two apparent Dirac points is much bigger than the gaps visible in each of the Dirac cones.

The Ni(111)-graphene system is $C_{3 v}$ symmetric, and the $d_{z^{2}}$ orbitals of the Ni atoms directly underneath graphene sublattice $A$ hybridize strongest with sublattice $A$ carbon atoms. By symmetry, the Ni orbitals of $E$ symmetry $\left(d_{x y}\right.$, $d_{x z}, d_{y z}$, and $\left.d_{x^{2}-y^{2}}\right)$ cannot hybridize with the graphene sublattice $A$ but only with sublattice $B$. Thus, the Ni $d_{z^{2}}$ orbitals and the Ni orbitals of $E$ symmetry play the role of boron and nitrogen, respectively, in the effective bilayer model, Eq. (2), which comes from dynamic hybridization in the more elaborated model. The symmetry arguments and the DFT calculations suggest a Hamiltonian

$$
H=\left(\begin{array}{cccc}
0 & v_{\mathrm{C}}\left(k_{x}+i k_{y}\right) & V^{\prime} & 0 \\
v_{\mathrm{C}}\left(k_{x}-i k_{y}\right) & 0 & 0 & V^{\prime \prime} \\
V^{\prime \dagger} & 0 & H_{\mathrm{Ni}-A A} & H_{\mathrm{Ni}-A E} \\
0 & V^{\prime \prime \dagger} & H_{\mathrm{Ni}-A E}^{\dagger} & H_{\mathrm{Ni}-E E}
\end{array}\right)
$$



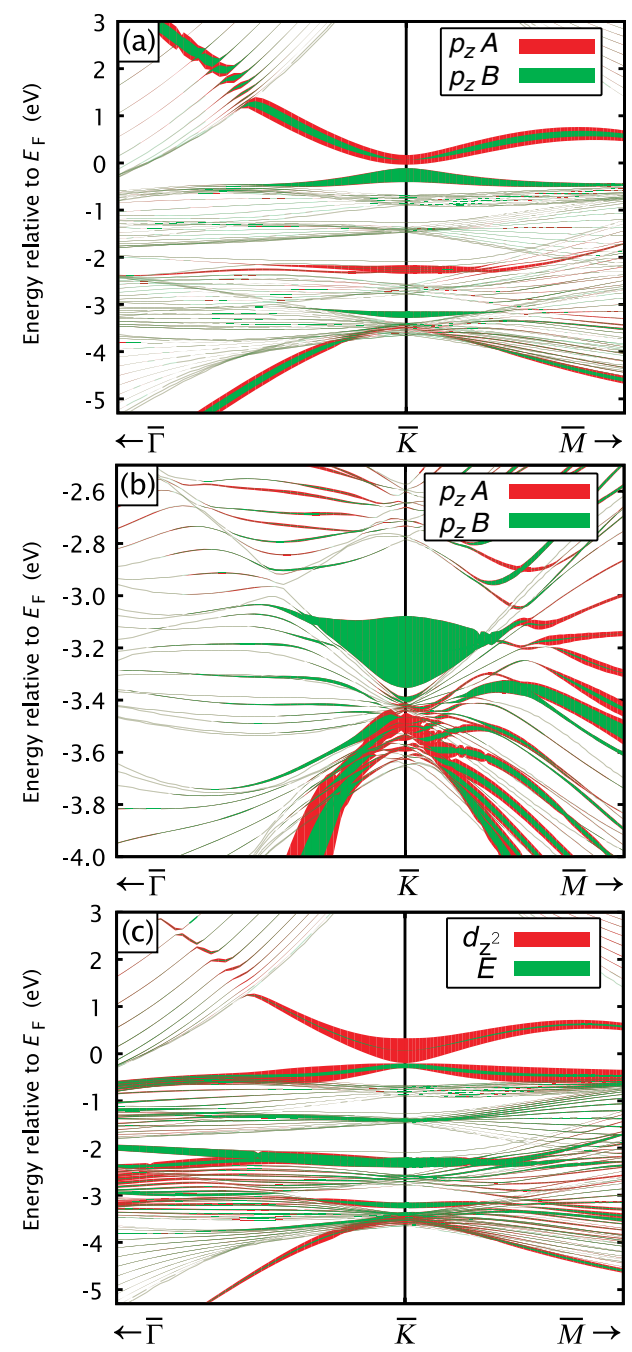

FIG. 7. Dynamic hybridization in the band structure of graphene on $\mathrm{Ni}(111)$. The thickness of the lines indicates the band character. (a),(b) Thickness corresponds to the carbon- $p_{z}$ contribution in sublattice $A$ (directly above $\mathrm{Ni}$, red lines) and sublattice $B$ (no $\mathrm{Ni}$ directly underneath, green lines). (c) Thickness corresponds to contributions from the $d$ orbitals of the topmost $\mathrm{Ni}$ atom, where $E=\left(d_{x y}, d_{x z}, d_{y z}, d_{x^{2}-y^{2}}\right)$. In all panels, only the majority spin is shown. The minority spin is qualitatively the same.

describing graphene on $\mathrm{Ni}(111)$ through the coupling $V^{\prime}$ between the Ni $d_{z^{2}}$ orbitals ( $A$ symmetry) and graphene sublattice $A$ and $V^{\prime \prime}$ between the $E$-type orbitals and sublattice $B$. The Hamiltonian (3) is formally already very close to Eq. (2). The major difference from Eq. (2) is that the Ni block in Eq. (3) is still a high-dimensional Hamiltonian encoding a continuum of bands coming from projecting out the $\mathbf{k}_{z}$ crystal momentum of bulk $\mathrm{Ni}$ and that $V^{\prime}\left(V^{\prime \prime}\right)$ are operators (block matrices) instead of simple numbers.

To arrive at a minimal model, we integrate out all but two Ni bands. These two Ni bands are chosen to qualitatively represent the Ni band structure in an energy window of experimental interest, which is here around the lower
Dirac cone at $E \sim-3 \mathrm{eV}$. This integration leads to a hybridization self-energy $\Sigma(k, \omega)$ acting on the carbon $p_{z}$ electrons. Approximating the self-energy to be real and static, $\Sigma(k, \omega)=\operatorname{diag}\left(E_{\mathrm{C} 1}, E_{\mathrm{C} 2}, 0,0\right)$ leads then to an effective Hamiltonian

$$
H=\left(\begin{array}{cccc}
E_{\mathrm{C} 1} & v_{\mathrm{C}}\left(k_{x}+i k_{y}\right) & t^{\prime} & 0 \\
v_{\mathrm{C}}\left(k_{x}-i k_{y}\right) & E_{\mathrm{C} 2} & 0 & t^{\prime \prime} \\
t^{\prime} & 0 & E_{A A}(k) & E_{A E}(k) \\
0 & t^{\prime \prime} & E_{A E}^{*}(k) & E_{E E}(k)
\end{array}\right) .
$$

This Hamiltonian contains several gap-generating terms, e.g., due to $E_{\mathrm{C} 1}-E_{\mathrm{C} 2} \neq 0, E_{\mathrm{AA}}(k) \neq E_{E E}(k)$, or $\left(E_{\mathrm{C} 1}+\right.$ $\left.E_{\mathrm{C} 2}\right) \neq\left[E_{A A}(k)+E_{E E}(k)\right]$. However, these different gapgenerating terms do not simply add up but can partially cancel each other, as we already learned from the discussion of the simple model (2).

The energy difference $e W$ in Eq. (2) resembles a term leading to doping of the graphene bands. However, Eqs. (2) and (4) are effective models that are applicable only in the energy region around the lower Dirac point, and all bands entering these models are of hybrid Ni- $d$ and graphene- $\pi$ character. Thus, the effective model parameter $W=-4 \mathrm{~V}$ does not imply a correspondingly huge charge transfer from $\mathrm{Ni}$ to graphene but has to be understood as coming from the hybridization between the Ni $3 d$ orbitals and graphene and as entering the model as a hybridization self-energy term.

\section{SUMMARY}

Our results reveal that the structural and electronic properties of graphene chemisorbed on fcc $\mathrm{Ni}(111)$ and hcp Co(0001) substrates are in severe contradiction, based on current understanding. On the one hand, graphene grows in the asymmetric $\mathcal{A C}$ stacking configuration, which leads to strong breaking of sublattice symmetry. On the other hand, photoemission demonstrates that the bands of Dirac fermions remain fully intact, with linear dispersion preserved and with no band gap at the Dirac energy. With model calculations of very different degrees of complexity, we have demonstrated that effects that usually open a gap at the Dirac point can also have the adverse effect of closing it. In the particular case of graphene on $\mathrm{Ni}$ and $\mathrm{Co}$, dynamical hybridization is identified as the underlying mechanism.

\section{ACKNOWLEDGMENTS}

A. V. acknowledges helpful discussions with N. Sandler. This work was supported by SPP 1459 of the Deutsche Forschungsgemeinschaft. B. V. acknowledges support from the Research Foundation Flanders (FWO-Vlaanderen). B. T. and T. O. W. would like to thank the KITP at Santa Barbara for hospitality during the completion of this work. 


\section{APPENDIX A: METHODS}

Photoemission experiments have been conducted with the ARPES $1^{2}$ end station at the UE112-PGM2 beam line of BESSY II. STM results have been acquired with an Omicron STM instrument. Ni(111) and Co(0001) substrates have been prepared by deposition of 20 atomic layers of $\mathrm{Ni}(\mathrm{Co})$ on clean $\mathrm{W}(110)$, with subsequent annealing at $750 \mathrm{~K}$. Graphene has been grown by CVD [20] using propylene [23]. The structural quality of the samples has been tested with LEED and STM. For Ni(111), CVD results in a perfect $p(1 \times 1)$ graphene structure without chiral dislocations. We have never seen any deterioration of the threefold symmetry of STM images [44] for varying conditions of the STM tip. For Co(0001), the CVD growth of graphene is less trivial. It always results in a small amount of additional rotational domains [23], but the ARPES and STM data discussed for graphene on Co refer to the $\mathrm{R} 0^{\circ}$ phase that is in registry with the Co substrate. ARPES measurements have been performed at room temperature and low temperature, as indicated in the figures.

[1] P. R. Wallace, The Band Theory of Graphite, Phys. Rev. 71, 622 (1947).

[2] K. S. Novoselov, A. K. Geim, S. V. Morozov, D. Jiang, M. I. Katsnelson, I. V. Grigorieva, S. V. Dubonos, and A. A. Firsov, Two-Dimensional Gas of Dirac Fermions in Graphene, Nature (London) 438, 197 (2005).

[3] M. I. Katsnelson, K. S. Novoselov, and A. K. Geim, Chiral Tunnelling and the Klein Paradox in Graphene, Nat. Phys. 2, 620 (2006).

[4] J.L. Mañes, F. Guinea, and María A.H. Vozmediano, Existence and Topological Stability of Fermi Points in Multilayered Graphene, Phys. Rev. B 75, 155424 (2007).

[5] T. Appelquist, D. Nash, and L.C. R. Wijewardhana, Critical Behavior in $(2+1)$-Dimensional QED, Phys. Rev. Lett. 60, 2575 (1988).

[6] Y. W. Son, M. L. Cohen, and S. G. Louie, Energy Gaps in Graphene Nanoribbons, Phys. Rev. Lett. 97, 216803 (2006).

[7] B. Trauzettel, D. V. Bulaev, D. Loss, and G. Burkard, Spin Qubits in Graphene Quantum Dots, Nat. Phys. 3, 192 (2007).

[8] R. Balog, B. Jørgensen, L. Nilsson, M. Andersen, E. Rienks, M. Bianchi, M. Fanetti, E. Lægsgaard, A. Baraldi, S. Lizzit, Z. Sljivancanin, F. Besenbacher, B. Hammer, T. G. Pedersen, Ph. Hofmann, and L. Hornekær, Bandgap Opening in Graphene Induced by Patterned Hydrogen Adsorption, Nat. Mater. 9, 315 (2010).

[9] T. Ohta, A. Bostwick, Th. Seyller, K. Horn, and E. Rotenberg, Controlling the Electronic Structure of Bilayer Graphene, Science 313, 951 (2006).

[10] D. P. DiVincenzo and E. J. Mele, Self-Consistent EffectiveMass Theory for Intralayer Screening in Graphite Intercalation Compounds, Phys. Rev. B 29, 1685 (1984).

[11] G. Giovannetti, P. A. Khomyakov, G. Brocks, P. J. Kelly, and J. van der Brink, Substrate-Induced Band Gap in Graphene on Hexagonal Boron Nitride: Ab Initio Density Functional Calculations, Phys. Rev. B 76, 073103 (2007).

[12] A. Bostwick, T. Ohta, J. L. McChesney, K. V. Emtsev, Th. Seyller, K. Horn, and E. Rotenberg, Symmetry Breaking in Few Graphene Layer Films, New J. Phys. 9, 385 (2007).

[13] S. Y. Zhou, D. A. Siegel, A. V. Fedorov, F. El Gabaly, A. K. Schmid, A. H. Castro Neto, D.-H. Lee, and A. Lanzara, Origin of the Energy Bandgap in Epitaxial Graphene, Nat. Mater. 7, 259 (2008).

[14] V. M. Karpan, P. A. Khomyakov, A. A. Starikov, G. Giovannetti, M. Zwierzycki, M. Talanana, G. Brocks, J. van den Brink, and P. J. Kelly, Theoretical Prediction of Perfect Spin Filtering at Interfaces between Close-Packed Surfaces of Ni or Co and Graphite or Graphene, Phys. Rev. B 78, 195419 (2008).

[15] A. Grüneis and D. V. Vyalikh, Tunable Hybridization between Electronic States of Graphene and a Metal Surface, Phys. Rev. B 77, 193401 (2008).

[16] S. Rusponi, M. Papagno, P. Moras, S. Vlaic, M. Etzkorn, P. M. Sheverdyaeva, D. Pacilé, H. Brune, and C. Carbone, Highly Anisotropic Dirac Cones in Epitaxial Graphene Modulated by an Island Superlattice, Phys. Rev. Lett. 105, 246803 (2010).

[17] Y.-M. Lin, C. Dimitrakopoulos, K. A. Jenkins, D. B. Farmer, H.-Y. Chiu, A. Grill, and Ph. Avouris, 100-GHz Transistors from Wafer-Scale Epitaxial Graphene, Science 327, 662 (2010).

[18] L. Liao, Y.-C. Lin, M. Bao, R. Cheng, J. Bai, Y. Liu, Y. Qu, K.L. Wang, Y. Huang, and X. Duan, High-Speed Graphene Transistors with a Self-Aligned Nanowire Gate, Nature (London) 467, 305 (2010).

[19] G. Giovannetti, P. A. Khomyakov, G. Brocks, V. M. Karpan, J. van den Brink, and P. J. Kelly, Doping Graphene with Metal Contacts, Phys. Rev. Lett. 101, 026803 (2008).

[20] Y. Gamo, A. Nagashima, M. Wakabayashi, M. Terai, and C. Oshima, Atomic Structure of Monolayer Graphite Formed on Ni(111), Surf. Sci. 374, 61 (1997).

[21] A. Varykhalov, J. Sánchez-Barriga, A. M. Shikin, C. Biswas, E. Vescovo, A. Rybkin, D. Marchenko, and O. Rader, Electronic and Magnetic Properties of Quasifreestanding Graphene on Ni, Phys. Rev. Lett. 101, 157601 (2008).

[22] O. Rader, A. Varykhalov, J. Sánchez-Barriga, D. Marchenko, A. Rybkin, and A. M. Shikin, Is There a Rashba Effect in Graphene on 3d Ferromagnets?, Phys. Rev. Lett. 102, 057602 (2009).

[23] A. Varykhalov and O. Rader, Graphene Grown on Co (0001) Films and Islands: Electronic Structure and Its Precise Magnetization Dependence, Phys. Rev. B 80, 035437 (2009).

[24] A. Nagashima, N. Tejima, and C. Oshima, Electronic States of the Pristine and Alkali-Metal-Intercalated Monolayer Graphite/Ni(111) Systems, Phys. Rev. B 50, 17487 (1994).

[25] D. Eom, D. Prezzi, K. T. Rim, H. Zhou, M. Lefenfeld, S. Xiao, C. Nuckolls, M. S. Hybertsen, T. F. Heinz, and G. W. Flynn, Structure and Electronic Properties of Graphene Nanoislands on Co(0001), Nano Lett. 9, 2844 (2009).

[26] G. Bertoni, L. Calmels, A. Altibelli, and V. Serin, FirstPrinciples Calculation of the Electronic Structure and 
EELS Spectra at the Graphene/Ni(111) Interface, Phys. Rev. B 71, 075402 (2005).

[27] P. A. Khomyakov, G. Giovannetti, P. C. Rusu, G. Brocks, J. van den Brink, and P. J. Kelly, First-Principles Study of the Interaction and Charge Transfer between Graphene and Metals, Phys. Rev. B 79, 195425 (2009).

[28] P. Mallet, F. Varchon, C. Naud, L. Magaud, C. Berger, and J.-Y. Veuillen, Electron States of Mono- and Bilayer Graphene on SiC Probed by Scanning-Tunneling Microscopy, Phys. Rev. B 76, 041403(R) (2007).

[29] R. Rosei, M. De Crescenzi, F. Sette, C. Quaresima, A. Savoia, and P. Perfetti, Structure of Graphitic Carbon on Ni(111): A Surface Extended-Energy-Loss Fine-Structure Study, Phys. Rev. B 28, 1161 (1983).

[30] Y. Souzu and M. Tsukada, Electronic States and Scanning Tunneling Spectroscopy Image of Monolayer Graphite on a Nickel (111) Surface by the DV-X $\alpha$ Method, Surf. Sci. 326, 42 (1995).

[31] I. Hamada and M. Otani, Comparative van der Waals Density-Functional Study of Graphene on Metal Surfaces, Phys. Rev. B 82, 153412 (2010).

[32] Yu. Dedkov, A. M. Shikin, V. K. Adamchuk, S. L. Molodtsov, C. Laubschat, A. Bauer, and G. Kaindl, Intercalation of Copper underneath a Monolayer of Graphite on Ni(111), Phys. Rev. B 64, 035405 (2001).

[33] E. N. Voloshina, A. Generalov, M. Weser, S. Böttcher, K. Horn, and Yu.S. Dedkov, Structural and Electronic Properties of the Graphene/Al/Ni(111) Intercalation System, New J. Phys. 13, 113028 (2011).

[34] Yu.S. Dedkov and M. Fonin, Electronic and Magnetic Properties of the Graphene-Ferromagnet Interface, New J. Phys. 12, 125004 (2010).

[35] E. L. Shirley, L. J. Terminello, A. Santoni, and F.J. Himpsel, Brillouin-Zone-Selection Effects in Graphite
Photoelectron Angular Distributions, Phys. Rev. B 51, 13614 (1995).

[36] B. Sachs, T. O. Wehling, M. I. Katsnelson, and A.I. Lichtenstein, Adhesion and Electronic Structure of Graphene on Hexagonal Boron Nitride Substrates, Phys. Rev. B 84, 195414 (2011).

[37] N. Kharche and S. K. Nayak, Quasiparticle Band Gap Engineering of Graphene and Graphone on Hexagonal Boron Nitride Substrate, Nano Lett. 11, 5274 (2011).

[38] A. Grüneis, K. Kummer, and D. V. Vyalikh, Dynamics of Graphene Growth on a Metal Surface: A Time-Dependent Photoemission Study, New J. Phys. 11, 073050 (2009) and references therein.

[39] S. Nie, A. L. Walter, N.C. Bartelt, E. Starodub, A. Bostwick, E. Rotenberg, and K.F. McCarty, Growth from Below: Graphene Bilayers on $\operatorname{Ir}(111)$, ACS Nano 5, 2298 (2011).

[40] A. Varykhalov, M. R. Scholz, Timur K. Kim, and O. Rader, Effect of Noble-Metal Contacts on Doping and Band Gap of Graphene, Phys. Rev. B 82, 121101(R) (2010).

[41] G. Kresse and J. Hafner, Norm-Conserving and Ultrasoft Pseudopotentials for First-Row and Transition Elements, J. Phys. Condens. Matter 6, 8245 (1994).

[42] G. Kresse and D. Joubert, From Ultrasoft Pseudopotentials to the Projector Augmented-Wave Method, Phys. Rev. B 59, 1758 (1999).

[43] L. V. Dzemiantsova, M. Karolak, F. Lofink, A. Kubetzka, B. Sachs, K. von Bergmann, S. Hankemeier, T. O. Wehling, R. Frömter, H.P. Oepen, A. I. Lichtenstein, and R. Wiesendanger, Multiscale Magnetic Study of Ni(111) and Graphene on Ni(111), Phys. Rev. B 84, 205431 (2011).

[44] R. Zhao, Y. Zhang, T. Gao, Y. Gao, N. Liu, L. Fu, and Z. Liu, Scanning Tunneling Microscope Observations of Non-AB Stacking of Graphene on Ni Films, Nano Res. 4, 712 (2011). 